\title{
Tension Pneumothorax Complicating a Perforation of a Duodenal Ulcer during ERCP with Endoscopic Sphincterotomy
}

An 80-ycar-old Chinese woman underwent emergency endoscopic retrograde cholangiopancreatography and endoscopic sphincterotomy for a biliary stricture. Active ulceration was noted in the first part of the duodenum. Endoscopic manipulation was difficult due to duodenal scarring, and a pre-cut sphincterotomy was performed. Facial and cervical surgical emphysema rapidly developed. The patient became cyanosed, unconscious, and suffered respiratory arrest. She was intubated endotracheally and ventilated with $100 \%$ oxygen. Fluoroscopy confirmed a right pneumothorax (Figure 1) and a pneumoperitoneum (Figure 2).

A right thoracostomy was carried out, and re-expansion of the lung was observed fluoroscopically. A chest drain was inserted. At laparotomy, pneumoperitoneum was confirmed. A $1-\mathrm{cm}$ perforated duodenal ulcer was found. There was fibrinous exudate on the falciform ligament and the inferior surface of the left lobe of the liver. There was little soiling elsewhere, indicating that it was a recent perforation. No air or bile was found in the retroperitoneum. The ulcer was repaired with an omental patch, and a biliary stent was inserted endoscopically six weeks later.

Perforation during endoscopic sphincterotomy is a well recognized complication, and it is usually retroperitoneal (1). It can cause retroperitoneal air dissection, subcutaneous emphysema, and pneumothorax (2). Pneumothorax may also be associated with intraperitoneal perforations (3). However, intraperitoneal perforation complicating the procedure is very rare, and has only been reported in unusual circumstances (4). In the present patient, perforation occurred at the site of a duodenal ulcer, and not at the sphincterotomy. We believe that manipulation of the endoscope pulled the duodenum off the falciform ligament and reopened a recent, healing perforation. Air passed into the peritoneum and thence into the thorax, leading to tension pneumothorax and subcutaneous emphysema.

Patients with duodenal ulceration commonly undergo diagnostic upper gastrointestinal endoscopy. Although this may entail less endoscopic manipulation than endoscopic sphincterotomy, the risk of perforation and escape of insufflated air from the gastrointestinal tract remains. Tension pneumothorax should be considered if collapse occurs during endoscopic procedures.

\section{A. P. Morley', J. Y. W. Lau' ${ }^{2}$, R. J. Young'}

'Dept. of Anaesthesia and Intensive Care

${ }^{2}$ Dept. of Surgery, Prince of Wales Hospital, Sha Tin,

New Territories, Hong Kong

\section{References}

1. Cotton PB, Lehman G, Vennes J, et al. Endoscopic sphincterotomy complications and their management: an attempt at consensus. Gastrointest Endosc 1991; 37: 383 -93.

2. Gya D, Sali A, Angus D. Subcutaneous emphysema and pneumothorax following endoscopic sphincterotomy. Aust N Z J Surg 1989; 59: 900-2.

3. Lim A, Ling R, Diggory R, Copeland G. Perforation of an abdominal viscus associated with a pneumothorax. Br J Clin Pract 1992; 46: 141-2.

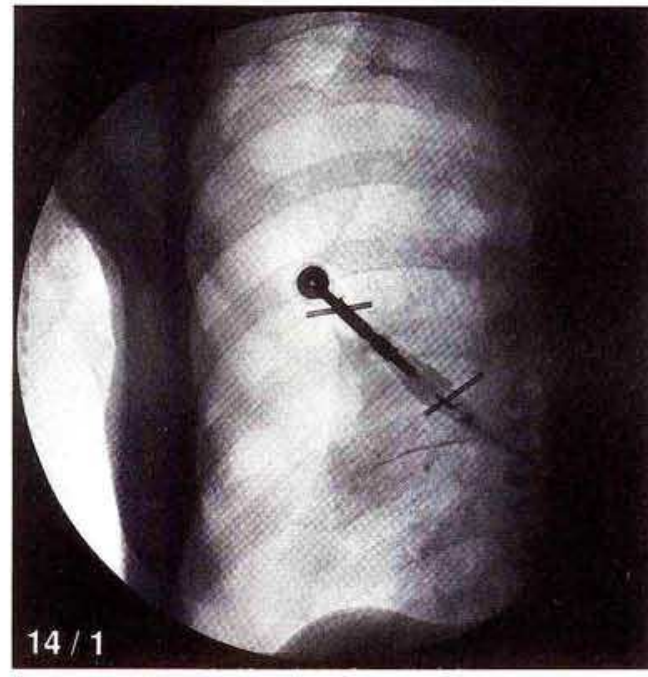

Figure 1: Anteroposterior chest radiograph, showing a right pneumothorax and subcutaneous emphysema.

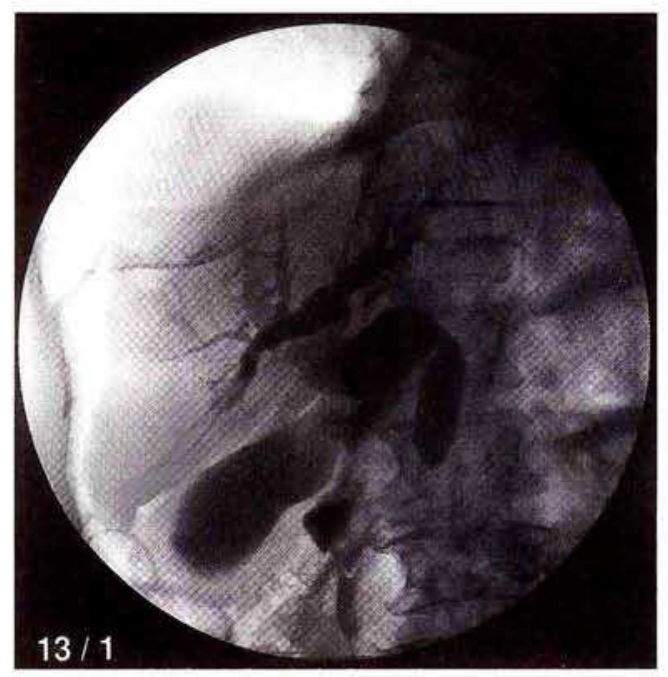

Figure 2: Anteroposterior abdominal radiograph, showing air under the right hemidiaphragm and residual contrast in the dilated biliary tree.

4. Bar-Meir S, Lang A, Shemesh E, et al. Pneumoperitoneum after insertion of endoscopic biliary stent for post-cholecystectomy biliary leak. Gastrointest Endosc 1993; 39: 818-20.

Corresponding Author

A. Morley, F.R.C.A.

Dept. of Anaesthesia and Intensive Care

Prince of Wales Hospital

Sha Tin

New Territories

Hong Kong

Fax: $+852-2637-2422$ 